Meta

Journal des tradlucteurs

Translators' Journal

\title{
BALLIU, C. (2002) : Les traducteurs transparents. La traduction en France à l'époque classique, Bruxelles, Éditions du Hazard, collection Traductologie, 240 pages.
}

\section{Hugo Marquant}

Volume 48, numéro 3, septembre 2003

Traduction et enseignement

Translation and teaching

URI : https://id.erudit.org/iderudit/007609ar

DOI : https://doi.org/10.7202/007609ar

Aller au sommaire du numéro

Éditeur(s)

Les Presses de l'Université de Montréal

ISSN

0026-0452 (imprimé)

1492-1421 (numérique)

Découvrir la revue

Citer ce compte rendu

Marquant, H. (2003). Compte rendu de [BALLIU, C. (2002) : Les traducteurs transparents. La traduction en France à l'époque classique, Bruxelles, Éditions du Hazard, collection Traductologie, 240 pages.] Meta, 48(3), 458-460.

https://doi.org/10.7202/007609ar d'utilisation que vous pouvez consulter en ligne.

https://apropos.erudit.org/fr/usagers/politique-dutilisation/ 
les besoins et exigences des utilisateurs. En la suivant dans cette réflexion sur la méthode lexicographique, on ne peut s'empêcher de conclure qu'elle gagne en lexicographie un très beau pari de la linguistique, auquel rêvent d'ailleurs toujours les linguistes générativistes et cognitivistes, celui d'accéder à l'universel par le truchement indispensable du particulier.

Éric Poirier

Université de Montréal, Montréal, Canada

Balliv, C. (2002): Les traducteurs transparents. La traduction en France à l'époque classique, Bruxelles, Éditions du Hazard, collection Traductologie, 240 pages.

À l'instar du $\mathrm{xv}^{\mathrm{e}}$ siècle italien (Quattrocento) et du $\mathrm{xvI}^{\mathrm{e}}$ espagnol (Siglo de Oro), le $\mathrm{XVII}^{\mathrm{e}}$ siècle français constitue sans conteste, tout en étant bien français, un des points forts absolus dans l'Histoire de la Culture Occidentale et de la Culture Universelle. Par sa vigueur extraordinaire (on ne l'appelle pas sans raison le Siècle des Querelles), par la diversité de ses excellences et surtout par la qualité exceptionnelle de ses représentants, le siècle de Louis XIV s'impose naturellement à tout qui se montre intéressé par l'étude diachronique d'un domaine particulier du savoir et du savoir-faire humain. Ce fut le temps où « un duc de la Rochefoucauld, l'auteur des Maximes, au sortir de la conversation d'un Pascal et d'un Arnauld, allait au Théâtre de Corneille» (Voltaire).

Dans son ouvrage sur la traduction en France à l'époque classique, le professeur C. Balliu poursuit un triple but: étudier la traduction du règne de Louis XIV dans un cadre de globalité politique, économique, religieuse, civile, juridique et artistique; réfléchir sur la traductologie en tant que dimension et développement linguistiques et littéraires (la traduction comme activité linguistique et littéraire) et, surtout, analyser la spécificité intellectuelle de la traduction (traductologie). En effet, on a pratiquement tout dit sur le $\mathrm{XVII}^{\mathrm{e}}$ siècle, sauf peut-être sur la traduction. Or, dans la réalité, tous les aspects de la traduction y ont été mis à l'honneur, la pratique d'abord, mais aussi l'enseignement, la réflexion et le débat.

Sur le plan théorique, le professeur Balliu, partisan convaincu de Georges Mounin (il lui emprunte notamment l'image de la transparence), considère que le débat littéralité vs fidélité est essentiellement et avant tout un faux débat. Il n'y a pas d'incompatibilité (antinomie) (p. 23) entre beauté et fidélité. Au Xvir siècle, écrivains et traducteurs se rencontrent et parlent le même langage. Que se soit à l'Académie ou dans les salons. Même l'opposition entre Académie et Jansénisme n'est qu'un leurre (p. 23-24).

L'approche du professeur Balliu est en même temps chronologique et thématique. En partant de Claude de Seyssel, le grand traducteur de Louis XII, d'Etienne Dolet, le «premier» traductologue français, de l'opposition Marot-du Bellay, du traducteur Vaugelas et de Jacques Amyot (le legs du passé) il débouche finalement sur Antoine Galland, le traducteur des Mille et une Nuits (1704) qui clôture le Grand Siècle. À l'intérieur de celui-ci l'auteur analyse attentivement l'état de la traduction par rapport à la qualité diachronique de la langue française, la préciosité, la réflexion traductologique, la querelle des Anciens et des Modernes, Port-Royal et l'enseignement ('’́cole des enfants de langues). 
Parmi les nombreux axes de réflexion qu'on peut trouver dans ce livre très bien présenté et rédigé, je voudrais en relever trois: le problème de la fidélité, l'importance de Port-Royal et l'amour de l'expérience orientaliste.

En traduction, le seul problème (ou s'agit-il tout simplement d'un élément définitoire?) est celui du rapport avec l'original (Mounin, 1955: Tous les arguments contre la traduction se résument en un seul: elle n'est pas l'original) (p. 17). Aussi la fidélité est-elle une des constantes de l'activité traduisante et traductologique de l'époque classique. Un des épisodes-clés de la querelle des Anciens et des Modernes n'est-il pas lié aux traductions d'Homère de Mme Dacier, savante helléniste, et M. Houdart de la Motte, qui ne savait même pas le grec! De même que le grand Arnauld intervient dans la polémique Boileau-Perrault, c'est Fénelon lui-même qui pacifia les esprits en 1715. Mais ce qui peut-être considéré sans conteste comme la manifestation suprême de la problématique mentionnée est la notion des Belles infidèles (Ménage), basée sur le phénomène de la transposition assortie d'une réflexion méthodologique ponctuelle: «si l'auteur était agréable à lire dans l'original, il doit l'être aussi en français» (p. 89). La fidélité conjuguée à l'esthétique du texte. Aussi les textes de Nicolas Perrot d'Ablancourt (Tacite, Lucien) ressemblent-ils plus à des «localisations» au sens actuel du terme qu'à des traductions.

Un deuxième point concerne l'importance de Port-Royal. À mon sentiment, le professeur Balliu a souligné avec raison le caractère brillant (les Provinciales de Pascal), universaliste (la Grammaire générale et raisonnée de Lancelot et Arnauld) et particulièrement riche (le «littéralisme» de Lemaistre de Sacy et les «infidélités» de Robert Arnauld d'Andilly; la réflexion traductologique-pédagogique d'Antoine Lemaistre et la pratique traduisante des «Messieurs») de l'expérience port-royaliste. Elle implique notamment une réflexion traductologique intéressante qui sera exploitée, complétée et améliorée par Gaspard de Tende et Pierre-Daniel Huet à partir de 1660. Le seul problème me semble finalement le fait d'accorder un statut à un ensemble qui, pour Antoine Arnauld, ne dépasse pas le niveau d'un fantôme.

Le troisième point a trait à une thématique qui intéresse plus particulièrement notre auteur: l'Orient et l'Occident. La querelle Galland-Mardrus autour de la traduction française des Mille et une nuits, recueil authentique de contes d'Orient, reflète d'une forme particulièrement explicite l'opposition entre ciblistes et sourciers. Elle clôture aussi le XVII ${ }^{e}$. Désormais, «[L]a beauté sera remplacée dans l'ordre de priorités par la spéculation philosophique, qui sacrifiera la délicatesse de l'écriture au maniement de l'idée» (p. 215); «le héros [...] [renonce] à l'introspection, à la complicité intime et secrète» (p. 216).

En conclusion, le moins que l'on puisse affirmer est que le professeur Balliu a réalisé pleinement ses objectifs. Parmi les grands mérites de son étude nous pouvons mentionner: la définition de la traduction comme produit sociologiquement déterminé d'une époque (p. 170), le lien entre les traducteurs et les gens de lettres (traduction et littérature), la sensibilité linguistique du traducteur (la langue française) et la valeur référentielle de la traductologie.

Mais surtout, notre auteur a réussi à échafauder une compétence référentielle sur l'ensemble du xvii et de la traductologie classique en particulier qui lui a permis de formuler un certain nombre d'hypothèses réussies et intéressantes, et surtout de ne pas tomber dans le piège du catalogue froid et inexpressif et de l'érudition embarras- 
sante (concentrée dans les notes). Le livre de C. Balliu se lit avec le même enthousiasme que celui de l'auteur et nous l'en remercions chaleureusement.

Hugo Marquant

Institut Libre Marie Haps, Bruxelles, Belgique

E. Steiner et C. Yallop (2001) (eds): Exploring Translation and Multilingual Text Production: Beyond Content, Berlin/New York, Mouton de Gruyter, coll. «Text, Translation, Computational Processing», 336 p.

Paru en 2001 aux Éditions Mouton de Gruyter dans la collection «Text, Translation, Computational Processing», l'ouvrage dirigé par Erich Steiner et Colin Yallop regroupe neuf articles qui s'interrogent sur la traduction multilingue, l'enseignement de la traduction, les outils informatiques d'aide aux langagiers et les rapports qu'entretiennent la linguistique et la traduction. Dans un texte d'introduction fouillé et méthodique, les deux directeurs de l'ouvrage justifient d'abord la pertinence du livre, publié dans cette collection et chez cet éditeur en particulier en raison de la filiation méthodologique qui les unit; en effet, l'éditeur et les directeurs s'intéressent aux modèles linguistiques actuels et insistent sur les développements récents en recherche traductionnelle. Steiner et Yallop présentent ensuite l'approche privilégiée, résolument tournée vers la réalité de la traduction, autrement dit «la vraie vie» des traducteurs et des traductologues, le souci de la profession étant au cour de leurs préoccupations. Enfin, ils mettent l'accent sur l'originalité du format (nous y reviendrons en conclusion).

Il importe de préciser que cet ouvrage traite principalement de la notion de sens, par opposition à celle de contenu. Les directeurs et les collaborateurs du livre souhaitent dépasser la notion de contenu en ce qu'elle constitue une barrière à l'appréhension du sens dans une perspective multidimensionnelle et stratifiée. En outre, la théorie fonctionnelle du langage de Michael Halliday - figure centrale du livre, comme le précisent Steiner et Yallop en introduction - sera reprise et analysée, non seulement par Halliday lui-même, mais par d'autres collaborateurs de l'ouvrage, une initiative qui montre la cohérence interne de ce collectif.

L’ouvrage est divisé en trois parties de longueurs inégales, intitulées respectivement: «Theoritical Orientation», «Modeling Translation» et «Working with Translation and Multilingual Texts: Computational and Didactic Projects», qui constitue la partie la plus courte (78 pages au total, contre 125 pages en moyenne pour chacune des deux autres). Toutefois, la valeur ne résidant pas dans la quantité mais dans la qualité, cette dernière partie, malgré sa minceur, est tout aussi chargée que les deux premières. Mais revenons à la première qui comporte trois articles aux visées théoriques. Celui de Michael Halliday interroge la qualité ou encore la valeur d'une traduction dépendant si on l'observe d'un point de vue linguistique ou traductionnel. L’article qui suit, signé par Michael Gregory et intitulé «What can Linguistics Learn from Translation?», présente d'abord la traduction en tant qu'activité humaine, donc possible. L'auteur s'appuie sur la traduction biblique afin de montrer le rôle important assumé par la mise en contexte et la formulation dans la réception d'un texte traduit. Enfin, Christian Matthiessen, dans «The Environments of Translation » 